$\mathrm{J}$ o u r n a l of

Mathematics

and Applications

JMA No 44, pp 19-34 (2021)

\title{
Extremal Solutions to a Coupled System of Nonlinear Fractional Differential Equations with $\psi$-Caputo Fractional Derivatives
}

\author{
Choukri Derbazi, Zidane Baitiche, Mouffak Benchohra and \\ John R. Graef
}

\begin{abstract}
Using the well-known monotone iterative technique together with the method of upper and lower solutions, the authors investigate the existence of extremal solutions to a class of coupled systems of nonlinear fractional differential equations involving the $\psi$-Caputo derivative with initial conditions. As applications of this work, two illustrative examples are presented.
\end{abstract}

AMS Subject Classification: 34A08, 26A33.

Keywords and Phrases: $\psi$-Caputo fractional derivative; Coupled system; Extremal solutions; Monotone iterative technique; Upper and lower solutions.

\section{Introduction}

Recently, more and more attention has been given to the subject of fractional differential and integral equations due to their importance in applications in various branches of applied science and engineering; see, for example, [18, 26, 27, 31, 33, 34, 35]. For basic facts in the fractional calculus, we refer to the books [20, 28, 30, 45]. Almeida [8] introduced a new and general fractional derivative called the $\psi$-Caputo fractional derivative and extended the work of several researchers [19, 20, 25]. Additional details and properties of this fractional derivative can be found in $[8,9,10,11,12]$. Other important qualitative properties such as existence, uniqueness, and stability of solutions of various fractional differential problems can be found in the papers $[4,5,6,7,21,39,40,41]$; see also $[1,2,3,45,46]$.

COPYRIGHT (C) by Publishing House of Rzeszów University of Technology P.O. Box 85, 35-959 Rzeszów, Poland 
The monotone iterative technique combined with the method of upper and lower solutions has been used by several authors to gain the existence and uniqueness of extremal solutions to nonlinear fractional differential equations (see, for instance, $[13,14,15,16,22,23,24,36,37,38,42,43,44])$.

Motivated by the above results, our goal is to extend the results in the recent paper by Derbazi et al. [15] by considering a more general system with the $\psi$-Caputo derivative. In [15], the authors considered the initial value problem

$$
\left\{\begin{array}{l}
{ }^{c} \mathbb{D}_{a^{+}}^{\alpha ; \psi} x(t)=f(t, x(t)), \quad t \in \mathrm{J}:=[a, b] \\
x(a)=a^{*}
\end{array}\right.
$$

where ${ }^{c} \mathbb{D}_{a^{+}}^{\alpha ; \psi}$ is the $\psi$-Caputo fractional derivative of order $\alpha \in(0,1], f \in C(\mathrm{~J} \times \mathbb{R})$, and $a^{*} \in \mathbb{R}$. They were interested in the existence and uniqueness of extremal solutions to $(1)$.

To the best of our knowledge, there are no known results on the existence of extremal solutions to systems of initial value problems for nonlinear fractional differential equations containing $\psi$-Caputo derivatives via the monotone iterative technique. As a result, we aim to fill this gap in the literature and contribute to enriching this area of research.

Here, we examine the existence of extremal solutions to the nonlinear coupled system

$$
\left\{\begin{array}{ll}
{ }^{c} \mathbb{D}_{a^{+}}^{\alpha ; \psi} x(t)=f_{1}(t, x(t), y(t)), & x(a)=x_{a}, \\
{ }^{c} \mathbb{D}_{a^{+}}^{\alpha ; \psi} y(t)=f_{2}(t, y(t), x(t)), & y(a)=y_{a},
\end{array} \quad t \in \mathrm{J}:=[a, b],\right.
$$

where ${ }^{c} \mathbb{D}_{a^{+}}^{\alpha ; \psi}$ is the $\psi$-Caputo fractional derivative of order $\alpha \in(0,1], f_{1}, f_{2} \in C(\mathrm{~J} \times$ $\left.\mathbb{R}^{2}, \mathbb{R}\right)$, and $x_{a}, y_{a} \in \mathbb{R}$ with $x_{a} \leq y_{a}$.

The structure of this paper is as follows. In Section 2, we introduce definitions and preliminary results that will be needed to prove our main results. In Section 3 , we apply the monotone iterative procedure and the method of upper and lower solutions to prove the existence of extremal solutions to the problem (2). In Section 4 , we present two examples to illustrate the applicability of our results.

\section{Preliminaries}

In this section, we introduce some notations and definitions from the fractional calculus and present preliminary results needed in our proofs.

Let $J=[a, b], 0<a<b<\infty$, be a finite interval and $\psi:[a, b] \rightarrow \mathbb{R}$ be an increasing function with $\psi^{\prime}(t) \neq 0$ for all $t \in[a, b]$.

We begin by defining $\psi$-Riemann-Liouville fractional integrals and derivatives. In what follows, $\Gamma(\cdot)$ is the (Euler's) Gamma function given by

$$
\Gamma(\alpha)=\int_{0}^{+\infty} t^{\alpha-1} e^{-t} \mathrm{dt}, \quad \alpha>0,
$$

and [ $\cdot]$ will denote the greatest integer function. 
Definition 2.1. [8, 20] For $\alpha>0$, the left-sided $\psi$-Riemann-Liouville fractional integral of order $\alpha$ of an integrable function $x: \mathrm{J} \longrightarrow \mathbb{R}$ with respect to the increasing differentiable function $\psi: \mathrm{J} \longrightarrow \mathbb{R}$ with $\psi^{\prime}(t) \neq 0$ for all $t \in \mathrm{J}$ is defined as

$$
\mathbb{I}_{a^{+}}^{\alpha ; \psi} x(t)=\frac{1}{\Gamma(\alpha)} \int_{a}^{t} \psi^{\prime}(s)(\psi(t)-\psi(s))^{\alpha-1} x(s) \mathrm{ds} .
$$

Definition 2.2. [8] Let $n \in \mathbb{N}$ and let $\psi, x \in C^{n}(\mathrm{~J}, \mathbb{R})$ be two functions such that $\psi$ is increasing with $\psi^{\prime}(t) \neq 0$ for all $t \in \mathrm{J}$. The left-sided $\psi$-Riemann-Liouville fractional derivative of a function $x$ of order $\alpha$ is defined by

$$
\begin{aligned}
\mathbb{D}_{a^{+}}^{\alpha ; \psi} x(t) & =\left(\frac{1}{\psi^{\prime}(t)} \frac{d}{d t}\right)^{n} \mathbb{I}_{a^{+}}^{n-\alpha ; \psi} x(t) \\
& =\frac{1}{\Gamma(n-\alpha)}\left(\frac{1}{\psi^{\prime}(t)} \frac{d}{d t}\right)^{n} \int_{a}^{t} \psi^{\prime}(s)(\psi(t)-\psi(s))^{n-\alpha-1} x(s) \mathrm{d} s
\end{aligned}
$$

where $n=[\alpha]+1$.

Definition 2.3. [8] Let $n \in \mathbb{N}$ and let $\psi, x \in C^{n}(\mathrm{~J}, \mathbb{R})$ be two functions such that $\psi$ is increasing and $\psi^{\prime}(t) \neq 0$ for all $t \in \mathrm{J}$. The left-sided $\psi$-Caputo fractional derivative of $x$ of order $\alpha$ is defined by

$$
{ }^{c} \mathbb{D}_{a^{+}}^{\alpha ; \psi} x(t)=\mathbb{I}_{a^{+}}^{n-\alpha ; \psi}\left(\frac{1}{\psi^{\prime}(t)} \frac{d}{d t}\right)^{n} x(t),
$$

where $n=[\alpha]+1$ for $\alpha \notin \mathbb{N}, n=\alpha$ for $\alpha \in \mathbb{N}$.

To simplify notation, we will use the abbreviated symbol

$$
x_{\psi}^{[n]}(t)=\left(\frac{1}{\psi^{\prime}(t)} \frac{d}{d t}\right)^{n} x(t) .
$$

From the above definition, it is clear that

$$
{ }^{c} \mathbb{D}_{a^{+}}^{\alpha ; \psi} x(t)= \begin{cases}\int_{a}^{t} \frac{\psi^{\prime}(s)(\psi(t)-\psi(s))^{n-\alpha-1}}{\Gamma(n-\alpha)} x_{\psi}^{[n]}(s) \mathrm{ds}, & \text { if } \alpha \notin \mathbb{N}, \\ x_{\psi}^{[n]}(t), & \text { if } \alpha \in \mathbb{N} .\end{cases}
$$

Lemma 2.1. [10, 20] Let $\alpha, \beta>0$ and $u \in C(\mathrm{~J}, \mathbb{R})$. Then for each $t \in \mathrm{J}$, we have:

(1) $\mathbb{I}_{a^{+}}^{\alpha ; \psi} \mathbb{I}_{a^{+}}^{\beta ; \psi} u(t)=\mathbb{I}_{a^{+}}^{\alpha+\beta ; \psi} u(t)$,

(2) ${ }^{c} \mathbb{D}_{a^{+}}^{\alpha ; \psi} \mathbb{I}_{a^{+}}^{\alpha ; \psi} x(t)=x(t)$,

(3) $\mathbb{I}_{a^{+}}^{\alpha ; \psi}(\psi(t)-\psi(a))^{\beta-1}=\frac{\Gamma(\beta)}{\Gamma(\beta+\alpha)}(\psi(t)-\psi(a))^{\beta+\alpha-1}$,

(4) ${ }^{c} \mathbb{D}_{a^{+}}^{\alpha ; \psi}(\psi(t)-\psi(a))^{\beta-1}=\frac{\Gamma(\beta)}{\Gamma(\beta-\alpha)}(\psi(t)-\psi(a))^{\beta-\alpha-1}$, 
(5) ${ }^{c} \mathbb{D}_{a^{+}}^{\alpha ; \psi}(\psi(t)-\psi(a))^{k}=0$, for all $k \in\{0, \ldots, n-1\}, n \in \mathbb{N}$.

Next, we recall the composition rules for fractional $\psi$-integrals and $\psi$-derivatives.

Lemma 2.2. [10] Let $\alpha>0$. If $x \in C^{n}(\mathrm{~J}, \mathbb{R})$ and $n-1<\alpha<n$, then

$$
\mathbb{I}_{a^{+}}^{\alpha ; \psi} \mathbb{D}_{a^{+}}^{\alpha ; \psi} x(t)=x(t)-\sum_{k=0}^{n-1} \frac{x_{\psi}^{[k]}(a)}{k !}[\psi(t)-\psi(a)]^{k}
$$

for all $t \in \mathrm{J}$.

Definition 2.4 ([17]). The Mittag-Leffler functions of one and two parameters are defined respectively as

$$
\mathbb{E}_{\alpha}(z)=\sum_{k=0}^{\infty} \frac{z^{k}}{\Gamma(\alpha k+1)}, \quad z \in \mathbb{R} \text { and } \alpha>0,
$$

and

$$
\mathbb{E}_{\alpha, \beta}(z)=\sum_{k=0}^{\infty} \frac{z^{k}}{\Gamma(\alpha k+\beta)}, \quad \alpha, \beta>0 \text { and } z \in \mathbb{R} .
$$

It is clear that $\mathbb{E}_{1,1}(z)=\mathbb{E}_{1}(z)=e^{z}$.

\section{Main Results}

In this section, we present the existence of extremal solutions to the system (2). The arguments are based on the monotone iterative technique combined with the method of upper and lower solutions. We begin by defining what we mean by a solution to (2).

Definition 3.1. By a solution of problem (2) we mean a pair of functions $(x, y) \in$ $C(\mathrm{~J}, \mathbb{R}) \times C(\mathrm{~J}, \mathbb{R})$ that satisfies the system

$$
\left\{\begin{array}{l}
{ }^{c} \mathbb{D}_{a^{+}}^{\alpha ; \psi} x(t)=f_{1}(t, x(t), y(t)), \\
{ }^{c} \mathbb{D}_{a^{+}}^{\alpha ; \psi} y(t)=f_{2}(t, y(t), x(t)),
\end{array}\right.
$$

on $\mathrm{J}$ with the initial conditions

$$
\left\{\begin{array}{l}
x(a)=x_{a} \\
y(a)=y_{a}
\end{array}\right.
$$

To prove the existence of solutions to the problem (2), we need the following lemma. 
Lemma 3.1. [15] Let $\alpha \in(0,1]$ be fixed, $\lambda \in \mathbb{R}$, and $h \in C(\mathrm{~J}, \mathbb{R})$. Then, the linear problem

$$
\left\{\begin{array}{l}
{ }^{c} \mathbb{D}_{a^{+}}^{\alpha \psi} x(t)+\lambda x(t)=h(t), t \in \mathrm{J}:=[a, b], \\
x(a)=x_{a},
\end{array}\right.
$$

has a unique solution given by

$$
\begin{aligned}
x(t)= & x_{a} \mathbb{E}_{\alpha, 1}\left(-\lambda(\psi(t)-\psi(a))^{\alpha}\right) \\
& +\int_{a}^{t} \psi^{\prime}(s)(\psi(t)-\psi(s))^{\alpha-1} \mathbb{E}_{\alpha, \alpha}\left(-\lambda(\psi(t)-\psi(s))^{\alpha}\right) h(s) \mathrm{ds},
\end{aligned}
$$

where $\mathbb{E}_{\alpha, \beta}(\cdot)$ is the two-parametric Mittag-Leffer function defined in (4).

Remark 3.1. Note that if $\lambda=0$ in Lemma 3.1, we do not need the Mittag-Leffler function to compute the solution of the linear problem, and in fact, the unique explicit solution of (5) is given by

$$
x(t)=x_{a}+\mathbb{I}_{a^{+}}^{\alpha ; \psi} h(t) .
$$

As a consequence of Lemma 3.1, we have the following result that will be useful in proving our main theorem.

Lemma 3.2. Let $\alpha \in(0,1]$ be fixed, $\lambda, \mu \in \mathbb{R}$, and $h, g \in C(\mathrm{~J}, \mathbb{R})$. Then the associated linear initial value problem

$$
\left\{\begin{array}{ll}
c \mathbb{D}_{a}^{\alpha ; \psi} x(t)+\lambda x(t)+\mu y(t)=h(t), & x(a)=x_{a}, \\
c \mathbb{D}_{a^{+}}^{\alpha ;} y(t)+\lambda y(t)+\mu x(t)=g(t), & y(a)=y_{a},
\end{array} \quad t \in \mathrm{J}:=[a, b],\right.
$$

has a unique solution in $C(\mathrm{~J}, \mathbb{R}) \times C(\mathrm{~J}, \mathbb{R})$.

Proof. Let

$$
x(t)=\frac{u(t)+v(t)}{2} \text { and } y(t)=\frac{u(t)-v(t)}{2} .
$$

Using (8), we have

$$
\left\{\begin{array}{l}
{ }^{c} \mathbb{D}_{a^{+}}^{\alpha ; \psi} u(t)+(\lambda+\mu) u(t)=(h+g)(t), t \in \mathrm{J}:=[a, b], \\
u(a)=u_{a}=x_{a}+y_{a}
\end{array}\right.
$$

and

$$
\left\{\begin{array}{l}
{ }^{c} \mathbb{D}_{a^{+}}^{\alpha ; \psi} v(t)+(\lambda-\mu) v(t)=(h-g)(t), t \in \mathrm{J}:=[a, b], \\
v(a)=v_{a}=x_{a}-y_{a} .
\end{array}\right.
$$

By Lemma 3.1, we know that (9) and (10) have a unique solution $u \in C(\mathrm{~J}, \mathbb{R})$ and $v \in C(\mathrm{~J}, \mathbb{R})$, respectively, that can be expressed as

$$
\begin{aligned}
u(t)= & u_{a} \mathbb{E}_{\alpha}\left(-(\lambda+\mu)(\psi(t)-\psi(a))^{\alpha}\right) \\
& +\int_{a}^{t} \psi^{\prime}(s)(\psi(t)-\psi(s))^{\alpha-1} \mathbb{E}_{\alpha, \alpha}\left(-(\lambda+\mu)(\psi(t)-\psi(s))^{\alpha}\right)(h+g)(s) \mathrm{ds}, \\
v(t)= & v_{a} \mathbb{E}_{\alpha}\left(-(\lambda-\mu)(\psi(t)-\psi(a))^{\alpha}\right) \\
& +\int_{a}^{t} \psi^{\prime}(s)(\psi(t)-\psi(s))^{\alpha-1} \mathbb{E}_{\alpha, \alpha}\left(-(\lambda-\mu)(\psi(t)-\psi(s))^{\alpha}\right)(h-g)(s) \mathrm{ds} .
\end{aligned}
$$


Consequently, the linear system (8) has a unique solution $(x, y)$.

Next we present two important comparison results that will play important roles in the proof of our main result.

First, we state the following lemma that was proven in [15, Lemma 5].

Lemma 3.3 (Comparison result). Let $\alpha \in(0,1]$ be fixed and $\tau \in \mathbb{R}$. If $\theta \in C(\mathrm{~J}, \mathbb{R})$ satisfies

$$
\left\{\begin{array}{l}
{ }^{c} \mathbb{D}_{a^{+}}^{\alpha ; \psi} \theta(t) \geq-\tau \theta(t), \quad t \in[a, b] \\
\theta(a) \geq 0
\end{array}\right.
$$

then $\theta(t) \geq 0$ for all $t \in \mathrm{J}$.

Based on the above lemma, we develop a new inequality involving the $\psi$-Caputo fractional derivative.

Lemma 3.4 (Comparison result). Let $\alpha \in(0,1]$ be fixed and $\lambda, \mu \in \mathbb{R}$ with $\mu \geq 0$. If $\rho, \nu \in C(\mathrm{~J}, \mathbb{R})$ satisfy

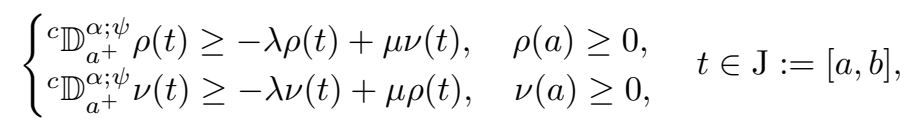

then $\rho(t) \geq 0, \nu(t) \geq 0$ for all $t \in \mathrm{J}$.

Proof. Let $\theta(t)=\rho(t)+\nu(t)$ for $t \in \mathrm{J}$. Then from (12),

$$
\left\{\begin{array}{l}
{ }^{c} \mathbb{D}_{a^{+}}^{\alpha ; \psi} \theta(t) \geq-(\lambda-\mu) \theta(t), \quad t \in \mathrm{J}:=[a, b] \\
\theta(a) \geq 0 .
\end{array}\right.
$$

From Lemma 3.3, we obtain $\theta(t) \geq 0, t \in \mathrm{J}$, which implies that

$$
\rho(t)+\nu(t) \geq 0 \text { for } t \in \mathrm{J} .
$$

Next, we will show that $\rho(t) \geq 0$ and $\nu(t) \geq 0$ for all $t \in \mathrm{J}$. In fact, from (12) and (13), we have

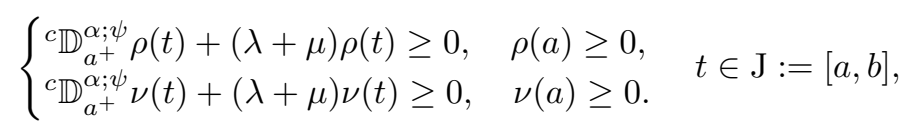

It follows from inequalities (14) and Lemma 3.3 that

$$
\rho(t) \geq 0 \quad \text { and } \quad \nu(t) \geq 0, \quad t \in \mathrm{J} .
$$


Lemma 3.5. Assume that $\left\{z_{n}(t)\right\}$ be a family of continuous functions on J satisfying

$$
\left\{\begin{array}{l}
{ }^{c} \mathbb{D}_{a^{+}}^{\alpha ; \psi} z_{n}(t)=f\left(t, z_{n}(t)\right), \quad t \in \mathrm{J}:=[a, b], \\
z_{n}(a)=z_{a}
\end{array}\right.
$$

for $n>0$ and where $\left|f\left(t, z_{n}(t)\right)\right| \leq M$ (M independent of $\left.n\right)$ for $t \in \mathrm{J}$. Then, the family $\left\{z_{n}(t)\right\}$ is equicontinuous on $\mathrm{J}$.

Proof. According to Remark 3.1, the integral representation of (15) is given by

$$
z_{n}(t)=z_{a}+\int_{a}^{t} \frac{\psi^{\prime}(s)(\psi(t)-\psi(s))^{\alpha-1}}{\Gamma(\alpha)} f\left(s, z_{n}(s)\right) \mathrm{d} s .
$$

For any $t_{1}, t_{2} \in \mathrm{J}$ with $a<t_{1}<t_{2}<b$, from (16) we have

$$
\begin{aligned}
\left|z_{n}\left(t_{2}\right)-z_{n}\left(t_{1}\right)\right| \leq & \int_{a}^{t_{1}} \frac{\psi^{\prime}(s)\left[\left(\psi\left(t_{1}\right)-\psi(s)\right)^{\alpha-1}-\left(\psi\left(t_{2}\right)-\psi(s)\right)^{\alpha-1}\right]}{\Gamma(\alpha)} \mid f\left(s, x_{n}(s) \mid \mathrm{ds}\right. \\
& +\int_{t_{1}}^{t_{2}} \frac{\psi^{\prime}(s)\left(\psi\left(t_{2}\right)-\psi(s)\right)^{\alpha-1}}{\Gamma(\alpha)}\left|f\left(s, x_{n}(s)\right)\right| \mathrm{ds} \\
\leq & \frac{M}{\Gamma(\alpha+1)}\left[\left(\psi\left(t_{1}\right)-\psi(a)\right)^{\alpha}+2\left(\psi\left(t_{2}\right)-\psi\left(t_{1}\right)\right)^{\alpha}-\left(\psi\left(t_{2}\right)-\psi(a)\right)^{\alpha}\right] \\
\leq & \frac{2 M}{\Gamma(\alpha+1)}\left(\psi\left(t_{2}\right)-\psi\left(t_{1}\right)\right)^{\alpha} .
\end{aligned}
$$

As $t_{2} \rightarrow t_{1}$, the right-hand side of the above inequality tends to zero independently of $\left\{z_{n}\right\}$. Hence, the family $\left\{z_{n}(t)\right\}$ is equicontinuous on $\mathrm{J}$.

Now, we are ready to establish our main theorem.

Theorem 3.1. Let the functions $f_{1}, f_{2} \in C(\mathrm{~J} \times \mathbb{R} \times \mathbb{R}, \mathbb{R})$. In addition, assume that:

$\left(H_{1}\right)$ There exist $x_{0}, y_{0} \in C(\mathrm{~J}, \mathbb{R})$ with $x_{0}(t) \leq y_{0}(t)$ for $t \in \mathrm{J}$ such that

$$
\begin{aligned}
& \left\{\begin{array}{l}
{ }^{c} \mathbb{D}_{a^{+}}^{\alpha ; \psi} x_{0}(t) \leq f_{1}\left(t, x_{0}(t), y_{0}(t)\right), \\
x_{0}(a) \leq x_{a},
\end{array}\right. \\
& \left\{\begin{array}{l}
{ }^{c} \mathbb{D}_{a^{+}}^{\alpha ; \psi} y_{0}(t) \geq f_{2}\left(t, y_{0}(t), x_{0}(t)\right), \\
y_{0}(a) \geq y_{a}
\end{array}\right.
\end{aligned}
$$

$\left(H_{2}\right)$ There exist constants $\lambda, \mu \in \mathbb{R}$ with $\mu \geq 0$ such that

$$
\begin{aligned}
& f_{1}(t, x, y)-f_{1}(t, \tilde{x}, \tilde{y}) \geq-\lambda(x-\tilde{x})-\mu(y-\tilde{y}), \\
& f_{2}(t, y, x)-f_{2}(t, \tilde{y}, \tilde{x}) \leq-\lambda(y-\tilde{y})-\mu(x-\tilde{x}),
\end{aligned}
$$

where $x_{0} \leq \tilde{x} \leq x \leq y_{0}, x_{0} \leq y \leq \tilde{y} \leq y_{0}$, and

$$
f_{2}(t, y, x)-f_{1}(t, x, y) \geq-\lambda(y-x)-\mu(x-y),
$$

for $x_{0} \leq x \leq y \leq y_{0}$. 
Then the system (2) has an extremal solution $\left(x^{*}, y^{*}\right) \in\left[x_{0}, y_{0}\right] \times\left[x_{0}, y_{0}\right]$. Moreover, there exist monotone iterative sequences $\left\{x_{n}\right\}_{n \in \mathbb{N}}$ and $\left\{y_{n}\right\}_{n \in \mathbb{N}} \subset\left[x_{0}, y_{0}\right]$ that converge uniformly to $x^{*}$ and $y^{*}$ respectively, where $\left\{x_{n}\right\}_{n \in \mathbb{N}}$ and $\left\{y_{n}\right\}_{n \in \mathbb{N}}$ are defined by

$$
x_{n+1}(t)=\frac{u_{n+1}(t)+v_{n+1}(t)}{2}, \quad y_{n+1}(t)=\frac{u_{n+1}(t)-v_{n+1}(t)}{2},
$$

with

$$
\begin{aligned}
u_{n+1}(t)= & \left(x_{a}+y_{a}\right) \mathbb{E}_{\alpha}\left(-(\lambda+\mu)(\psi(t)-\psi(a))^{\alpha}\right) \\
+ & \int_{a}^{t} \psi^{\prime}(s)(\psi(t)-\psi(s))^{\alpha-1} \mathbb{E}_{\alpha, \alpha}\left(-(\lambda+\mu)(\psi(t)-\psi(s))^{\alpha}\right) \\
& \times\left(f_{1}\left(s, x_{n}(s), y_{n}\right)+f_{2}\left(s, y_{n}(s), x_{n}\right)+(\lambda+\mu)\left(x_{n}(s)+y_{n}(s)\right)\right) \mathrm{d} \mathrm{s}, \\
v_{n+1}(t)= & \left(x_{a}-y_{a}\right) \mathbb{E}_{\alpha}\left(-(\lambda-\mu)(\psi(t)-\psi(a))^{\alpha}\right) \\
+ & \int_{a}^{t} \psi^{\prime}(s)(\psi(t)-\psi(s))^{\alpha-1} \mathbb{E}_{\alpha, \alpha}\left(-(\lambda-\mu)(\psi(t)-\psi(s))^{\alpha}\right) \\
& \quad \times\left(f_{1}\left(s, x_{n}(s), y_{n}\right)-f_{2}\left(s, y_{n}(s), x_{n}\right)+(\lambda-\mu)\left(x_{n}(s)-y_{n}(s)\right)\right) \mathrm{ds},
\end{aligned}
$$

and

$$
x_{0}(t) \leq x_{1}(t) \leq \cdots \leq x_{n}(t) \leq \cdots \leq y_{n}(t) \leq \cdots \leq y_{1}(t) \leq y_{0}(t), \quad t \in \mathrm{J} .
$$

Proof. For any $x_{0}(t), y_{0}(t) \in C(\mathrm{~J}, \mathbb{R})$, we consider the linear system

$$
\left\{\begin{array}{l}
{ }^{c} \mathbb{D}_{a^{+}}^{\alpha ; \psi} x_{n+1}(t)=f_{1}\left(t, x_{n}(t), y_{n}(t)\right)-\lambda\left(x_{n+1}(t)-x_{n}(t)\right)-\mu\left(y_{n+1}(t)-y_{n}(t)\right), \quad t \in \mathrm{J}, \\
\quad x_{n+1}(a)=x_{a}, \\
{ }^{c} \mathbb{D}_{a^{+}}^{\alpha ; \psi} y_{n+1}(t)=f_{2}\left(t, y_{n}(t), x_{n}(t)\right)-\lambda\left(y_{n+1}(t)-y_{n}(t)\right)-\mu\left(x_{n+1}(t)-x_{n}(t)\right), \quad t \in \mathrm{J}, \\
y_{n+1}(a)=y_{a} .
\end{array}\right.
$$

By Lemma 3.1, we know that $(21)$ has a unique solution in $C(\mathrm{~J}, \mathbb{R}) \times C(\mathrm{~J}, \mathbb{R})$ that are defined by (19). We complete the proof of the theorem through the following three steps.

Step 1: The sequences $\left\{x_{n}(t)\right\}$ and $\left\{y_{n}(t)\right\}$ satisfy the relation

$$
x_{n}(t) \leq x_{n+1}(t) \leq y_{n+1}(t) \leq y_{n}(t), \quad n=0,1,2, \cdots, t \in \mathrm{J}
$$

First, we prove that

$$
x_{0}(t) \leq x_{1}(t) \leq y_{1}(t) \leq y_{0}(t), \quad t \in \mathrm{J} .
$$

Set $\rho(t)=x_{1}(t)-x_{0}(t)$ and $\nu(t)=y_{0}(t)-y_{1}(t)$. From $(21)$ and $\left(H_{1}\right)$, we see that

$$
\left\{\begin{array}{ll}
{ }^{c} \mathbb{D}_{a+}^{\alpha ; \psi} \rho(t) \geq-\lambda \rho(t)+\mu \nu(t), & \rho(a) \geq 0, \\
{ }^{c} \mathbb{D}_{a^{+}}^{\alpha ; \psi} \nu(t) \geq-\lambda \nu(t)+\mu \rho(t), & \nu(a) \geq 0 .
\end{array} \quad t \in \mathrm{J}:=[a, b],\right.
$$


By Lemma 3.4, $\rho(t) \geq 0$ and $\nu(t) \geq 0$, for all $t \in \mathrm{J}$. That is, $x_{0}(t) \leq x_{1}(t) . y_{1}(t) \leq$ $y_{0}(t), t \in \mathrm{J}$.

Now, let $\theta(t)=y_{1}(t)-x_{1}(t)$. By $(21)$ and $\left(H_{2}\right)$, we have

$$
\begin{aligned}
{ }^{c} \mathbb{D}_{a^{+}}^{\alpha ; \psi} \theta(t)= & { }^{c} \mathbb{D}_{a^{+}}^{\alpha ; \psi} y_{1}(t)-{ }^{c} \mathbb{D}_{a^{+}}^{\alpha ; \psi} x_{1}(t) \\
= & f_{2}\left(t, y_{0}(t), x_{0}(t)\right)-f_{1}\left(t, x_{0}(t), y_{0}(t)\right)+\lambda\left(y_{0}(t)-x_{0}(t)\right) \\
& +\mu\left(x_{0}(t)-y_{0}(t)\right)-(\lambda-\mu)\left(y_{1}(t)-x_{1}(t)\right) \\
\geq & -\lambda\left(y_{0}(t)-x_{0}(t)\right)-\mu\left(x_{0}(t)-y_{0}(t)\right)+\lambda\left(y_{0}(t)-x_{0}(t)\right) \\
& +\mu\left(x_{0}(t)-y_{0}(t)\right)-(\lambda-\mu) \theta(t) \\
= & -(\lambda-\mu) \theta(t) .
\end{aligned}
$$

Since, $\theta(a)=y_{1}(a)-x_{1}(a)=y_{a}-x_{a} \geq 0$. By Lemma 3.3, we obtain $x_{1}(t) \leq y_{1}(t), t \in$ J.

Next, we show that $x_{1}(t)$ and $y_{1}(t)$ satisfy inequalities (17) and (18), respectively. Since $x_{0}$ and $y_{0}$ are respective solutions of (17) and (18), it follows that

$$
\left\{\begin{aligned}
{ }^{c} \mathbb{D}_{a^{+}}^{\alpha ; \psi} x_{1}(t) & =f_{1}\left(t, x_{0}(t), y_{0}(t)\right)-\lambda\left(x_{1}(t)-x_{0}(t)\right)-\mu\left(y_{1}(t)-y_{0}(t)\right) \\
& \leq f_{1}\left(t, x_{1}(t), y_{1}(t)\right) \\
x_{1}(a) & \leq x_{a}
\end{aligned}\right.
$$

and

$$
\left\{\begin{aligned}
{ }^{c} \mathbb{D}_{a^{+}}^{\alpha ; \psi} y_{1}(t) & =f_{2}\left(t, y_{0}(t), x_{0}(t)\right)-\lambda\left(y_{1}(t)-y_{0}(t)\right)-\mu\left(x_{1}(t)-x_{0}(t)\right) \\
& \geq f_{2}\left(t, y_{1}(t), x_{1}(t)\right) \\
y_{1}(a) & \geq y_{a} .
\end{aligned}\right.
$$

Therefore, $x_{1}(t)$ and $y_{1}(t)$ satisfy the inequalities (17) and (18), respectively.

By the above arguments and mathematical induction, the relation (22) holds.

Step 2: The sequences $\left\{x_{n}\right\}$ and $\left\{y_{n}\right\}$ converge uniformly to their limit functions $x^{*}$ and $y^{*}$, respectively. By (20), the sequences $\left\{x_{n}\right\}$ and $\left\{y_{n}\right\}$ are uniformly bounded on J. From Lemma 3.5, the sequences $\left\{x_{n}\right\}$ and $\left\{y_{n}\right\}$ are equicontinuous on J. Hence by the Ascoli-Arzelà Theorem, there exist subsequences $\left\{x_{n_{k}}\right\}$ and $\left\{y_{n_{k}}\right\}$ that converge uniformly to $x^{*}$ and $y^{*}$, respectively, on J. This, together with the monotonicity of the sequences $\left\{x_{n}\right\}$ and $\left\{y_{n}\right\}$, implies

$$
\lim _{n \rightarrow \infty} x_{n}(t)=x^{*}(t) \text { and } \lim _{n \rightarrow \infty} y_{n}(t)=y^{*}(t),
$$

uniformly on $t \in \mathrm{J}$, and the limit functions $x^{*}, y^{*}$ satisfy the problem (2).

Step 3: System (2) has an extremal solution. Assume that $(x(t), y(t)) \in$ $\left[x_{0}(t), y_{0}(t)\right] \times\left[x_{0}(t), y_{0}(t)\right]$ be any solutions of system $(2)$. That is,

$$
\left\{\begin{array}{ll}
{ }^{c} \mathbb{D}_{a+}^{\alpha ; \psi} x(t)=f_{1}(t, x(t), y(t)), & x(a)=x_{a}, \\
{ }^{c} \mathbb{D}_{a^{+}}^{\alpha ; \psi} y(t)=f_{2}(t, y(t), x(t)), & y(a)=y_{a} .
\end{array} \quad t \in \mathrm{J}:=[a, b],\right.
$$


We need to prove that $x^{*} \leq x$ and $y \leq y^{*}$; we do so by using induction. Clearly, $x_{0}(t) \leq x(t)$ and $y(t) \leq y_{0}(t)$. Assume that for some $n \in \mathbb{N}$,

$$
x_{n}(t) \leq x(t) \quad \text { and } \quad y(t) \leq y_{n}(t), \quad t \in \mathrm{J}
$$

Let $\rho(t)=x(t)-x_{n+1}(t)$ and $\nu(t)=y_{n+1}(t)-y(t)$. From $(21)$ and $\left(H_{2}\right)$, we obtain

$$
\begin{aligned}
{ }^{c} \mathbb{D}_{a^{+}}^{\alpha ; \psi} \rho(t) & ={ }^{c} \mathbb{D}_{a^{+}}^{\alpha ; \psi} x(t)-{ }^{c} \mathbb{D}_{a^{+}}^{\alpha ; \psi} x_{n+1}(t) \\
& =f_{1}(t, x(t), y(t))-f_{1}\left(t, x_{n}(t), y_{n}\right)+\lambda\left(x_{n+1}(t)-x_{n}(t)\right) \\
& +\mu\left(y_{n+1}(t)-y_{n}(t)\right) \geq-\lambda\left(x(t)-x_{n}(t)\right)-\mu\left(y(t)-y_{n}(t)\right) \\
& +\lambda\left(x_{n+1}(t)-x_{n}(t)\right)+\mu\left(y_{n+1}(t)-y_{n}(t)\right) \\
& =-\lambda\left(x(t)-x_{n+1}(t)\right)-\mu\left(y(t)-y_{n+1}(t)\right)=-\lambda \rho(t)+\mu \nu(t)
\end{aligned}
$$

and

$$
\rho(a)=x(a)-x_{n+1}(a)=x_{a}-x_{a}=0 .
$$

Furthermore,

$$
\begin{aligned}
{ }^{c} \mathbb{D}_{a^{+}}^{\alpha ; \psi} \nu(t) & ={ }^{c} \mathbb{D}_{a^{+}}^{\alpha ; \psi} y_{n+1}(t)-{ }^{c} \mathbb{D}_{a^{+}}^{\alpha ; \psi} y(t) \\
& =f_{2}\left(t, y_{n}(t), x_{n}(t)\right)-f_{2}(t, y(t), x(t))-\lambda\left(y_{n+1}(t)-y_{n}(t)\right) \\
& -\mu\left(x_{n+1}(t)-x_{n}(t)\right) \geq \lambda\left(y(t)-y_{n}(t)\right)+\mu\left(x(t)-x_{n}(t)\right) \\
& -\lambda\left(y_{n+1}(t)-y_{n}(t)\right)-\mu\left(x_{n+1}(t)-x_{n}(t)\right) \\
& =-\lambda\left(y_{n+1}(t)-y(t)\right)+\mu\left(x(t)-x_{n+1}(t)\right)=-\lambda \nu(t)+\mu \rho(t)
\end{aligned}
$$

and

$$
\nu(a)=y_{n+1}(a)-y(a)=y_{a}-y_{a}=0
$$

Hence, the above argument yields

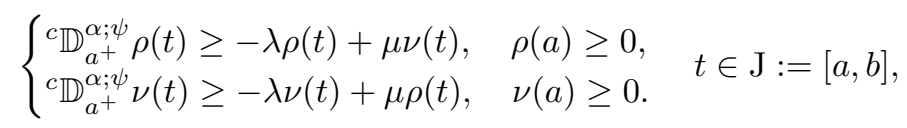

By Lemma 3.4, it follows that

$$
x_{n+1}(t) \leq x(t) \quad \text { and } \quad y(t) \leq y_{n+1}(t), t \in \mathrm{J}
$$

Therefore, (24) holds on J for all $n \in \mathbb{N}$. Taking the limit as $n \rightarrow \infty$ on both sides of (24), we get

$$
x^{*} \leq x, \quad y \leq y^{*} .
$$

Therefore $\left(x^{*}, y^{*}\right)$ is an extremal solution of system $(2)$ in $\left[x_{0}, y_{0}\right] \times\left[x_{0}, y_{0}\right]$. This completes the proof of the theorem 


\section{Examples}

In this section, we provide two examples to demonstrate the applicability of our results.

Example 4.1. Consider the fractional differential system

$$
\left\{\begin{array}{ll}
{ }^{c} \mathbb{D}_{0^{+}}^{0.5 ; \psi} x(t)=(t-x(t))^{2}-0.5 t y(t), & x(0)=0, \\
{ }^{c} \mathbb{D}_{0^{+}}^{0.5 ; \psi} y(t)=(t-y(t))^{2}-0.5 t x(t), & y(0)=0,
\end{array} \quad t \in[0,1],\right.
$$

where

$$
\alpha=0.5, a=0, b=1, \psi(t)=t, x_{a}=y_{a}=0
$$

and

$$
\left\{\begin{array}{l}
f_{1}(t, x, y)=(t-x)^{2}-0.5 t y, \\
f_{2}(t, y, x)=(t-y)^{2}-0.5 t x .
\end{array} \quad t \in[0,1],\right.
$$

Taking $x_{0}(t)=0$ and $y_{0}(t)=t$, we obtain

$$
\left\{\begin{array}{ll}
{ }^{c} \mathbb{D}_{0^{+}}^{0.5 ; \psi} x_{0}(t)=0 \leq 0.5 t^{2}=f_{1}\left(t, x_{0}(t), y_{0}(t)\right), & x_{0}(0)=0, \\
{ }^{c} \mathbb{D}_{0^{+}}^{0.5 ; \psi} y_{0}(t)=2 \sqrt{\frac{t}{\pi}} \geq 0=f_{2}\left(t, y_{0}(t), x_{0}(t)\right), & y_{0}(0)=0 .
\end{array} \quad t \in[0,1],\right.
$$

On the other hand, it is easily to verify that condition $\left(H_{2}\right)$ holds for $\lambda=2$ and $\mu=0$. It follows from Theorem 3.1 that the nonlinear fractional differential system (25) has an extremal solution $\left(x^{*}, y^{*}\right) \in[0, t] \times[0, t]$. Furthermore, we have the iterative sequences

$$
\begin{aligned}
& x_{n+1}(t)=\int_{0}^{t}(t-s)^{-0.5} \mathbb{E}_{0.5,0.5}(-2 \sqrt{t-s})\left(\left(s-x_{n}(s)\right)^{2}-s y_{n}(s)+2 x_{n}(s)\right) \mathrm{d} s, n \geq 0, \\
& y_{n+1}(t)=\int_{0}^{t}(t-s)^{-0.5} \mathbb{E}_{0.5,0.5}(-2 \sqrt{t-s})\left(\left(s-y_{n}(s)\right)^{2}-s x_{n}(s)+2 y_{n}(s)\right) \mathrm{d} s, n \geq 0 .
\end{aligned}
$$

Example 4.2. Consider the coupled system

$$
\left\{\begin{array}{ll}
{ }^{C H} \mathbb{D}_{1+}^{0.5 ; \psi} x(t)=2\left(\ln ^{2}(t)-x^{2}(t)\right)-\ln (t) y(t), & x(1)=0, \\
{ }^{C H} \mathbb{D}_{1^{+}}^{0.5 ; \psi} y(t)=2\left(\ln ^{2}(t)-y^{2}(t)\right)-\ln (t) x(t), & y(1)=0,
\end{array} \quad t \in[1, e]\right.
$$

where

$$
\alpha=0.5, a=1, b=e, \psi(t)=\ln (t), x_{a}=y_{a}=0,
$$

and

$$
\left\{\begin{array}{l}
f_{1}(t, x, y)=2\left(\ln ^{2}(t)-x^{2}\right)-\ln (t) y, \\
f_{2}(t, y, x)=2\left(\ln ^{2}(t)-y^{2}\right)-\ln (t) x .
\end{array} \quad t \in[1, e],\right.
$$

Taking $x_{0}(t)=0$ and $y_{0}(t)=\ln (t)$, it is not difficult to show that (H1) holds. Also, it can easily be verified that condition $\left(H_{2}\right)$ holds with $\lambda=4$ and $\mu=0$. Hence, the hypothesis of Theorem 3.1 are satisfied, and so the nonlinear fractional differential 
system (26) has an extremal solution $\left(x^{*}, y^{*}\right) \in[0, \ln (t)] \times[0, \ln (t)]$. Moreover, the monotone iterative sequences $\left\{x_{n}\right\}_{n \in \mathbb{N}},\left\{y_{n}\right\}_{n \in \mathbb{N}}$ can be obtained by

$$
\begin{aligned}
x_{n+1}(t) & =\int_{1}^{t}\left(\ln \frac{t}{s}\right)^{-0.5} \mathbb{E}_{0.5,0.5}\left(-4 \sqrt{\ln \frac{t}{s}}\right)\left(2\left(\ln ^{2}(s)-x_{n}^{2}(s)\right)\right. \\
& \left.-\ln (s) y_{n}(s)+4 x_{n}(s)\right) \frac{\mathrm{ds}}{\mathrm{s}}, n \geq 0 \\
y_{n+1}(t) & =\int_{1}^{t}\left(\ln \frac{t}{s}\right)^{-0.5} \mathbb{E}_{0.5,0.5}\left(-4 \sqrt{\ln \frac{t}{s}}\right)\left(2\left(\ln ^{2}(s)-y_{n}^{2}(s)\right)\right. \\
& \left.-\ln (s) x_{n}(s)+4 y_{n}(s)\right) \frac{\mathrm{ds}}{\mathrm{s}}, n \geq 0 .
\end{aligned}
$$

Acknowledgment. The authors wish to thank the two anonymous reviewers who carefully read the manuscript and made many helpful suggestions for improving the paper.

\section{References}

[1] S. Abbas, M. Benchohra, G.M. N'Guérékata, Topics in Fractional Differential Equations, Dev. Math., 27, Springer, New York, 2015.

[2] S. Abbas, M. Benchohra, G.M.N'Guérékata, Advanced Fractional Differential and Integral Equations, Nova Sci. Publ., New York, 2014.

[3] S. Abbas, M. Benchohra, J.R. Graef, J. Henderson, Implicit Fractional Differential and Integral Equations: Existence and Stability, De Gruyter, Berlin, 2018.

[4] S. Abbas, M. Benchohra, N. Hamidi, J. Henderson, Caputo-Hadamard fractional differential equations in Banach spaces, Fract. Calc. Appl. Anal. 21 (2018) $1027-1045$.

[5] S. Abbas, M. Benchohra, B. Samet, Y. Zhou, Coupled implicit Caputo fractional q-difference systems, Adv. Difference Equ., Paper No. 527 (2019) 1-19.

[6] R.P. Agarwal, M. Benchohra, S. Hamani, A survey on existence results for boundary value problems of nonlinear fractional differential equations and inclusions, Acta Appl. Math. 109 (2010) 973-1033.

[7] A. Aghajani, E. Pourhadi, J.J. Trujillo, Application of measure of noncompactness to a Cauchy problem for fractional differential equations in Banach spaces, Fract. Calc. Appl. Anal. 16 (2013) 962-977.

[8] R. Almeida, A Caputo fractional derivative of a function with respect to another function, Commun. Nonlinear Sci. Numer. Simul. 44 (2017) 460-481. 
[9] R. Almeida, Fractional differential equations with mixed boundary conditions, Bull. Malays. Math. Sci. Soc. 42 (2019) 1687-1697.

[10] R. Almeida, A.B. Malinowska, M.T.T. Monteiro, Fractional differential equations with a Caputo derivative with respect to a kernel function and their applications, Math. Meth. Appl. Sci. 41 (2018) 336-352.

[11] R. Almeida, A.B. Malinowska, T. Odzijewicz, Optimal leader-follower control for the fractional opinion formation model, J. Optim. Theory Appl. 182 (2019) 1171-1185.

[12] R. Almeida, M. Jleli, B. Samet, A numerical study of fractional relaxationoscillation equations involving $\psi$-Caputo fractional derivative, Rev. R. Acad. Cienc. Exactas Fís. Nat. Ser. A Mat. RACSAM 113 (2019) 1873-1891.

[13] M. Al-Refai, M. Ali Hajji, Monotone iterative sequences for nonlinear boundary value problems of fractional order, Nonlinear Anal. 74 (2011) 3531-3539.

[14] C. Chen, M. Bohner, B. Jia, Method of upper and lower solutions for nonlinear Caputo fractional difference equations and its applications, Fract. Calc. Appl. Anal. 22 (2019) 1307-1320.

[15] C. Derbazi, Z. Baitiche, M. Benchohra, A. Cabada, Initial value problem for nonlinear fractional differential equations with $\psi$-Caputo derivative via monotone iterative technique, Axioms 9 (2020) 57. DOI:10.3390/axioms9020057

[16] H. Fazli, H.G. Sun, S. Aghchi, Existence of extremal solutions of fractional Langevin equation involving nonlinear boundary conditions, Int. J. Comput. Math. 2020 (2020) 1-10.

[17] R. Gorenflo, A.A. Kilbas, F. Mainardi, S. V. Rogosin, Mittag-Leffler Functions, Related Topics and Applications, Springer, New York, 2014.

[18] R. Hilfer, Applications of Fractional Calculus in Physics, World Scientific, Singapore, 2000.

[19] F. Jarad, T. Abdeljawad, D. Baleanu, Caputo-type modification of the Hadamard fractional derivatives, Adv. Difference Equ. 2012 (142) (2012) 1-8.

[20] A.A. Kilbas, H.M. Srivastava, J.J. Trujillo, Theory and Applications of Fractional Differential Equations, North-Holland Mathematics Studies, Vol. 204, Elsevier, Amsterdam, 2006.

[21] K.D. Kucche, A. Mali, J. Vanterler da C. Sousa, On the nonlinear $\Psi$-Hilfer fractional differential equations. Comput. Appl. Math. 38 (2019) Art. 73, 1-25.

[22] K.D. Kucche, A.D. Mali, Initial time difference quasilinearization method for fractional differential equations involving generalized Hilfer fractional derivative, Comput. Appl. Math. 39, Paper No. 31 (2020) 1-33. 
[23] X. Lin, Z. Zhao, Iterative technique for a third-order differential equation with three-point nonlinear boundary value conditions, Electron. J. Qual. Theory Differ. Equ. 2016, Paper No. 12 (2016) 1-10.

[24] S. Liu, H. Li, Extremal system of solutions for a coupled system of nonlinear fractional differential equations by monotone iterative method, J. Nonlinear Sci. Appl. 9 (2016) 3310-3318.

[25] Y. Luchko, J.J. Trujillo, Caputo-type modification of the Erdélyi-Kober fractional derivative, Fract. Calc. Appl. Anal. 10 (2007) 249-267.

[26] F. Mainardi, Fractional Calculus and Waves in Linear Viscoelasticity, Imperial College Press, London, 2010.

[27] M. Metwali, On some qualitative properties of integrable solutions for Cauchytype problem of fractional order, J. Math. Appl. 40 (2017) 121-134.

[28] K.S. Miller, B. Ross, An Introduction to Fractional Calculus and Fractional Differential Equations, Wiley, New York, 1993.

[29] K.B. Oldham, Fractional differential equations in electrochemistry, Adv. Eng. Softw. 41 (2010) 9-12.

[30] I. Podlubny, Fractional Differential Equations, Academic Press, San Diego, 1999.

[31] J. Sabatier, O.P. Agrawal, J.A.T. Machado, Advances in Fractional CalculusTheoretical Developments and Applications in Physics and Engineering, Springer, Dordrecht, 2007.

[32] B. Samet, H. Aydi, Lyapunov-type inequalities for an anti-periodic fractional boundary value problem involving $\psi$-Caputo fractional derivative, J. Inequal. Appl. 2018, Paper No. 286 (2018) 1-11.

[33] W. El-Sayed, M. El-Borai, M. Metwali, N. Shemais, On the existence of continuous solutions of a nonlinear quadratic fractional integral equation, J. Advances Math. 19 (2020) 14-25.

[34] V.E. Tarasov, Fractional Dynamics, Nonlinear Physical Science, Springer, Heidelberg, 2010.

[35] V.E. Tarasov (Editor), Handbook of Fractional Calculus with Applications, Vol. 5, Applications in Physics, Part B, De Gruyter, Berlin, 2019.

[36] G. Wang, R.P. Agarwal, A. Cabada, Existence results and the monotone iterative technique for systems of nonlinear fractional differential equations, Appl. Math. Lett. 25 (2012) 1019-1024.

[37] G. Wang, W. Sudsutad, L. Zhang, J. Tariboon, Monotone iterative technique for a nonlinear fractional q-difference equation of Caputo type, Adv. Difference Equ., 2016, Paper No. 211 (2016) 1-11. 
[38] G. Wang, J. Qin, L. Zhang, D. Baleanu, Monotone iterative method for a nonlinear fractional conformable p-Laplacian differential system, Math. Meth. Appl. Sci. 2020, 1-11.

[39] J. Wang, L. Lv, Y. Zhou, Ulam stability and data dependence for fractional differential equations with Caputo derivative, Electron. J. Qual. Theory Differ. Equ. 2011 (63) (2011) 1-10.

[40] J. Wang, X. Li, E $E_{\alpha}$-Ulam type stability of fractional order ordinary differential equations, J. Appl. Math. Comput. 45 (2014) 449-459.

[41] J. Wang, Y. Zhang, Ulam-Hyers-Mittag-Leffler stability of fractional-order delay differential equations, Optimization 63 (2014) 1181-1190.

[42] N. Xu, W. Liu, Iterative solutions for a coupled system of fractional differentialintegral equations with two-point boundary conditions, Appl. Math. Comput. 244 (2014) 903-911.

[43] W. Yang, Monotone iterative technique for a coupled system of nonlinear Hadamard fractional differential equations, J. Appl. Math. Comput. 59 (2019) 585-596.

[44] S. Zhang, Monotone iterative method for initial value problem involving Riemann-Liouville fractional derivatives, Nonlinear Anal. 71 (2009) 2087-2093.

[45] Y. Zhou, Basic Theory of Fractional Differential Equations, World Scientific, Singapore, 2014.

[46] Y. Zhou, Fractional Evolution Equations and Inclusions: Analysis and Control, Elsevier, 2016.

\section{DOI: $10.7862 /$ rf.2021.2}

\section{Choukri Derbazi}

email: choukriedp@yahoo.com

ORCID: 0000-0003-2830-1027

Laboratory of Mathematics And Applied Sciences

University of Ghardaia

47000 Algeria

ALGERIA

Zidane Baitiche

email: baitichezidane19@gmail.com

ORCID: 0000-0003-4841-5398

Laboratory of Mathematics And Applied Sciences

University of Ghardaia 
47000 Algeria

ALGERIA

Mouffak Benchohra

email: benchohra@yahoo.com

ORCID: $\mathrm{xxxx}$

Laboratory of Mathematics

Djillali Liabes University of Sidi-Bel-Abbes

ALGERIA

John R. Graef

email: john-graef@utc.edu

ORCID: 0000-0002-8149-4633

Department of Mathematics

University of Tennessee at Chattanooga

Chattanooga

TN 37403

USA

Received 28.0\%.2020

Accepted 30.01.2021 